Arch. Tierz., Dummerstorf 42 (1999) 6, 593-600

Aus dem Institut fur Tierzucht und Tierverhalten der FAL, Mariensec' und der Züchtungszentrale Deutsches Hybridschwein GmbH, Laneburg ${ }^{2}$

ULRICH BAULAIN ${ }^{1}$ und HUBERT HENNE ${ }^{2}$

\title{
Variation des Fleischanteiles im Bauch von Schweinen aus Mutterlinien
}

\author{
Herrn Professor Dr. Peter Glodek zum 65. Geburtstag gewidmet
}

\begin{abstract}
Summary
Title of the paper: Variation of lean content in pig bellies of dam lines

Magnetic resonance imaging (MRI) was applied to determine the tissue composition of 157 bellies of castrated male pigs belonging to two dam-lines of the German Hybrid Pig Breeding Programme. All pigs were taken from the routine testing scheme. The analysis of carcass traits showed that pigs of line 03 were clearly leaner than those of line 01 . Only in backfat thickness no significant difference could be observed. For the belly, a clear difference of $7 \%$ was found in percentage of lean. Lean content of the belly was also estimated by means of various multiple regressions using carcass traits. Percentage of lean predicted by the official equation used in German performance testing on the one hand and by means of MRI on the other hand was different particularly in line 03 . A correlation of $r=0.67$ and $r=0.82$ between lean content determined by means of MRI and multiple regression was found for line 01 and line 03 , respectively. To study the variation between sires, variance components were estimated. Differences between sires were higher in lean content of the belly compared to backfat thickness, a trait which has been used in selection for a long time.
\end{abstract}

Key Words: pig, belly, body composition, magnetic resonance imaging

\section{Zusammenfassung}

Mit Hilfe des Magnet-Resonanz-Imagings wurde die gewebliche Zusammensetzung von insgesamt 157 Bäuchen von Börgen aus zwei Mutterlinien des Bundeshybridzuchtprogrammes bestimmt. Alle Tiere stammten aus der routinemäßigen Vollgeschwisterprüfung. Die Auswertung der Schlachtkörpermerkmale zeigte, daß die Tiere der Linie 03 fleischreicher waren als die der Linie 01, wobei in der Rückenspeckdicke keine signifikante Differenz festgestellt wurde. Dagegen ergab die Analyse des Fleischanteiles im Bauch einen Linienunterschied von 7\%Punkten. Eine Schätzung des Bauchfleischanteiles über Schlachtkörpermerkmale, die anhand verschiedener multipler Regressionen durchgefuhrt wurde, ergab unterschiedliche Werte. Der anhand der Gruber Formel geschätzte Bauchfleischanteil wich insbesondere in Linie 03 deutlich von dem mit MRI erfaßten ab. Die Korrelationen zwischen den auf diese Weise geschätzten Bauchfleischanteilen lagen für Linie 01 bei $r=0,67$ und für Linie 03 bei $r=0,82$. Öber eine gezielte Auswahl von Vätern und Nachkommen wurde versucht, den Vatereinfluß auf die erfaßten Merkmale zu schätzen. Die größte Varianz zwischen Vätern wurde im Merkmal Bauchfleischanteil beobachtet. Dagegen wiesen Merkmale wie die schon lange züchterisch bearbeitete Rückenspeckdicke eine deutlich geringere Vätervarianz auf.

Schlüsselwörter: Schwein, Bauch, Körperzusammensetzung, Magnet-Resonanz-Imaging 
1. Einleitung

Der Wert eines Schlachtkörpers wird in erster Linie durch seinen Fleischanteil bestimmt. Da die Vermarktung von Teilstücken immer mehr Bedeutung erlangt, werden zunehmend Informationen über die gewebliche Zusammensetzung dieser Teilstücke benötigt. Der Bauch weist im Vergleich zu Kotelett, Schinken und Schulter deutlich niedrigere Beziehungen zum Fleischanteil der Schlachthälfte auf (FEWSON et al., 1990). Bei der Bewertung auf Basis des geschätzten Fleischanteiles des gesamten Schlachtkörpers wird die Bauchqualität daher nur unzureichend erfaßt und sollte separat bewertet werden. Ein in der Leistungsprüfung gängiges Verfahren ist nach wie vor die subjektive Bonitierung des Bauchanschnittes in Höhe der 13./14. Rippe. Eine andere Möglichkeit ist die Schätzung des Fleischanteiles im Bauch über Muskel- und Fettmaße am Schlachtkörper. Im Jahr 1997 wurde die bis dahin in der Leistungsprüfung gültige Bonner Formel durch die Gruber Formel ersetzt (THOLEN et al., 1998). Auf einigen größeren Schlachthöfen kann der Fleischanteil von Teilstücken mittlerweile mit Hilfe des Autofom-Gerätes geschätzt werden (BRANSCHEID et al., 1997; BRONDUM et al., 1998). Ein bislang ausschließlich in der Forschung eingesetztes Verfahren ist das Magnet-Resonanz-Imaging (MRI), das die nicht invasive Aufnahme von Querschnittsbildern ermöglicht und eine sehr genaue Differenzierung zwischen und innerhalb von Weichgeweben liefert (BAULAIN, 1997). Das Ziel der vorliegenden Untersuchung bestand darin, den Fleischanteil von Schweinebäuchen aus Mutterlinien mit Hilfe des Magnet-Resonanz-Imagings zu erfassen und die Beziehungen zu den in der Leistungsprüfung erhobenen Schlachtkörpermaßen zu analysieren. Durch eine gezielte Auswahl von Vätern und Nachkommen sollte versucht werden, Hinweise auf die Vätervarianz zu erhalten.

\section{Material und Methode}

Für den im Zeitraum April bis August 1997 durchgeführten Versuch standen insgesamt 157 Börge aus den Mutterlinien 01 und 03 des Bundeshybridzuchtprogrammes zur Verfügung. Die Tiere stammten aus der Vollgeschwisterprüfung des BHZP, die zentral auf einem Masttestbetrieb erfolgte. Es wurden reine Halbgeschwistergruppen untersucht; die Väter der Tiere waren nicht verwandt. Nach Mastende wurden die Schweine auf dem Schlachthof in Lüneburg geschlachtet, wobei die in der Leistungsprüfung üblichen Schlachtmerkmale erhoben wurden. Die Tiere der Linie 01 hatten ein durchschnittliches Schlachtgewicht von $87,1 \mathrm{~kg}$ bei einer Standardabweichung von $4,1 \mathrm{~kg}$, die Schlachtkörper der Linie 03 wogen $88,4 \mathrm{~kg} \pm 4,5 \mathrm{~kg}$. Die nicht angeschnittene rechte Bauchhälfte wurde gekühlt per Kurier nach Mariensee befördert und einen Tag später im Magnet-Resonanz-Tomographen untersucht. Wamme und Gesäugeleiste waren entsprechend der DLG-Schnittführung (Ladenbauch) zuvor entfernt worden. Aus meßtechnischen Gründen mußten auch die Rippen extrahiert werden. Bei einer Scheibendicke von $8 \mathrm{~mm}$ und einem Scheibenabstand von $16 \mathrm{~mm}$ wurden je nach Länge der Bäuche bis zu 28 parallele Querschnittsbilder aufgenommen. In einer semi-automatischen Bildauswertung erfolgte eine Klassifizierung der einzelnen Bildpunkte als Mus- 
kel- oder Fettgewebe. Als Resultat ergaben sich Muskel- und Fettflächen für jedes Schnittbild. Aus der Summe der Flächen, multipliziert mit dem Scheibenabstand, wurden dann die Muskel- und Fettvolumina des gesamten Bauches berechnet. Die Eignung dieses Verfahrens zur Bestimmung des Fleischanteiles von Schweinebäuchen wurde von BAULAIN et al. (1998) nachgewiesen. Von der Auswertung wurden drei Tiere wegen unvollständiger Bildserien, drei Tiere aufgrund unvollständiger LP-Daten und ein Tier aufgrund eines großen Eiterherdes im Muskelgewebe des Bauches ausgeschlossen. Der detaillierte Versuchsumfang ist in Tabelle 1 angegeben.

Tabelle 1

Anzahl untersuchter Tiere (Number of animals investigated)

\begin{tabular}{cccccc}
\hline Linie & Anzahl Tiere & Anzahl Väter & Tiere/Vater & Min. & Max. \\
\hline 01 & 96 & 13 & 7,4 & 4 & 10 \\
03 & 54 & 14 & 3,9 & 3 & 7 \\
\hline
\end{tabular}

Die statistischen Analysen wurden mit Hilfe des SAS-Programmpaketes durchgeführt (SAS, 1989). Zur Untersuchung der Einflußfaktoren Linie und Eber wurde für alle Merkmale ein gemischtes Modell mit dem fixen Effekt Linie, dem zufälligen Effekt Eber innerhalb Linie und der Kovariablen Schlachtgewicht angewendet (Proc Mixed). Aus den Varianzkomponenten wurde die Intraklasskorrelation berechnet als $\mathrm{IKK}=\mathrm{V}_{\mathrm{S}} /\left(\mathrm{V}_{\mathrm{S}}+\mathrm{V}_{\mathrm{E}}\right)$. In Regressionsanalysen über das Gesamtmaterial und innerhalb der Linien wurde die Abhängigkeit des Fleischanteiles im Bauch von Schlachtkörpermaßen aus der Leistungsprüfung untersucht (Proc Reg).

\section{Ergebnisse}

Aus den in den Tabelle 2 dargestellten Ergebnissen der Varianzanalyse für die Schlachtkörpermerkmale wird ersichtlich, daß die Börge der Linie 03 deutlich magerer waren als die der Linie 01. Eine Ausnahme bildete nur das Merkmal Rückenspeckdicke, wo kein signifikanter Linienunterschied nachgewiesen werden konnte.

Tabelle 2

LSQ-Mittelwerte und Standardfehler von Schlachtkörpermerkmalen aus der Leistungsprüfung (LS means and standard errors of carcass traits from performance testing)

\begin{tabular}{lllll}
\hline Merkmal & \multicolumn{2}{c}{ Linie 01 } & \multicolumn{2}{c}{ Linie 03 } \\
& LSM & SEM & LSM & SEM \\
\hline MFA gesamt (FOM, \%) & $50,00^{\mathrm{a}}$ & 0,47 & $53,06^{\mathrm{b}}$ & 0,55 \\
& & & & \\
Rückenspeck (mm) & $26,52^{\mathrm{a}}$ & 0,40 & $25,38^{\mathrm{a}}$ & 0,48 \\
Seitenspeck (mm) & $24,96^{\mathrm{a}}$ & 0,69 & $20,58^{\mathrm{b}}$ & 0,75 \\
Fleischfläche (cm $\left.{ }^{2}\right)$ & $34,21^{\mathrm{a}}$ & 0,55 & $37,37^{\mathrm{b}}$ & 0,62 \\
Fettfläche $\left(\mathrm{cm}^{2}\right)$ & $19,78^{\mathrm{a}}$ & 0,43 & $17,22^{\mathrm{b}}$ & 0,51 \\
& & & & \\
Bauchpunkte & $4,99^{\mathrm{a}}$ & 0,11 & $5,52^{\mathrm{b}}$ & 0,12 \\
\hline a,b Mittelwerte unterscheiden sich signifikant bei unterschiedlichem Superscript (p<0,01)
\end{tabular}

Neben der Bestimmung des Bauchfleischanteiles mit Hilfe des MR-Imagings wurde eine Schätzung über Schlachtkörpermaße durchgeführt. Dabei kamen sowohl die Gruber- als auch die Bonner Formel zur Anwendung. Auch unter Berücksichtigung, 
daß der mit MRI bestimmte Fleischanteil die Einheit Vol.-\% aufweist, führten die unterschiedlichen Verfahren der Bestimmung des Fleischanteiles zu deutlich voneinander abweichenden Ergebnissen (Tab. 3). Mit allen Methoden wurde nachgewiesen, daß die Bäuche der Linie 03 magerer waren als die der Linie 01, aber während die Liniendifferenz nach MRI sieben Prozentpunkte betrug, waren es nach Gruber Formel weniger als zwei Prozentpunkte. Ebenso fiel auf, daß bei dem mit der Gruber Formel geschätzten Fleischanteil die Varianz in beiden Linien deutlich niedriger war als bei dem mit MRI erfaßten. Die Standardabweichungen betrugen für Linie 01 2,26\% gegenüber $4,36 \%$ und für Linie 03 2,99\% gegenüber 5,26\%. Der mit der Bonner Formel geschätzte Bauchfleischanteil ergab dagegen eine engere Übereinstimmung mit den Resultaten aus dem MR-Imaging.

Daraufhin wurden sowohl für das Gesamtmaterial als auch innerhalb der Linien Regressionsanalysen durchgeführt, um zu untersuchen, mit welcher Genauigkeit die Zielgröße Fleischanteil nach MRI anhand der in der Leistungsprüfung erfaßten Schlachtkörpermaße geschätzt werden kann. Für das Gesamtmaterial wurde folgende multiple Regression berechnet:

Fleischanteil im Bauch (\%) $=53,49+0,501 *$ Fleischfläche $\left(\mathrm{cm}^{2}\right)$

- 0,288* Rückenspeckdicke (mm)

$-0,429 *$ Seitenspeckdicke $(\mathrm{mm})$

$-0,330 *$ Speck über Rücken (mm)

Mit dieser Formel konnte $74 \%$ der Varianz im Bauchfleischanteil erklärt werden (Bestimmtheitsmaß); der Schätzfehler lag bei 2,93\%. Die Überprüfung dieser Formel innerhalb der Linien zeigte eine gute Eignung für beide Linien. Für die Linie 01 und 03 wurden mittlere Fleischanteile von $46,6 \%$ bzw. 51,6\% geschätzt. Damit wurde im Vergleich zu den Ergebnissen aus dem MR-Imaging (Tab. 3) der Fleischanteil in Linie 01 leicht über-, in Linie 03 dagegen leicht unterschätzt.

Tabelle 3

LSQ-Mittelwerte und Standardfehler des Fleischanteiles im Bauch bestimmt mit Hilfe des MR-Imagings und anhand von Schlachtkörpermaßen (LS means and standard errors for lean content in belly determined by MRI and from different carcass traits)

\begin{tabular}{lccrc}
\hline Merkmal & \multicolumn{2}{c}{ Linie 01 } & \multicolumn{2}{c}{ Linie 03 } \\
& LSM & SEM & LSM & SEM \\
\hline Gewicht des Bauches (kg) & $4,27^{\mathrm{a}}$ & 0,04 & $3,88^{\mathrm{b}}$ & 0,05 \\
MFA Bauch (MRI, Vol.-\%) & $45,71^{\star}$ & 0,87 & $52,75^{\mathrm{b}}$ & $\mathbf{0 , 9 3}$ \\
MFA Bauch (Gruber Formel, \%) & $53,23^{\mathrm{a}}$ & 0,39 & $55,67^{\mathrm{b}}$ & 0,44 \\
MFA Bauch (Bonner Formel, \%) & $45,36^{\mathrm{a}}$ & 0,69 & $49,07^{\mathrm{b}}$ & 0,80 \\
MFA Bauch (BHZP Formel, Vol.-\%) & $46,60^{\mathrm{a}}$ & 0,78 & $51,62^{\mathrm{b}}$ & 0,84 \\
\hline
\end{tabular}

$\mathrm{a}, \mathrm{b} \quad$ Mittelwerte unterscheiden sich signifikant bei unterschicdlichem Superscript $(p<0,01)$

Aus Tabelle 4 geht hervor, daß der mit FOM geschätzte Fleischanteil der Hälfte und der mit MRI bestimmte Fleischanteil des Bauches für beide Mutterlinien eine Korrelation von $r=0,7$ aufweist. Bei den Muskel- und Fettmaßen wurden für Linie 03 tendenziell höhere Korrelationen zum Fleischanteil des Bauches festgestellt als für Linie 01. Die subjektive Bewertung (Bonitierung) der Bäuche wies sehr unterschiedliche Be- 
zichungen zu dem mit MRI bestimmten Bauchfleischanteil auf. Während für Linie 01 eine Korrelation von $r=0,62$ gefunden wurde, ergab sich für Linie 03 eine Korrelation von $\mathrm{r}=0,21$.

Tabelle 4

Korrelationen zwischen dem Fleischanteil im Bauch und Schlachtkörpermerkmalen aus der Leistungsprüfung (Correlations between percentage of lean in belly and carcass traits from performance testing)

\begin{tabular}{lcc}
\hline & Linie 01 & Linie 03 \\
\hline FA gesamt (FOM) & 0,69 & 0,71 \\
Rückenspeck & $-0,62$ & $-0,67$ \\
Seitenspeck & $-0,55$ & $-0,81$ \\
Fleischfläche & 0,50 & 0,57 \\
Fettfläche & $-0,62$ & $-0,70$ \\
& & 0,21 \\
Bauchpunkte & 0,62 & 0 \\
\hline
\end{tabular}

In Tabelle 5 sind die Korrelationen zwischen den mit den verschiedenen Methoden bestimmten Fleischanteilen des Bauches aufgeführt. Innerhalb der Mutterlinie 03 lagen alle Korrelationen bei $\mathrm{r}>0,8$. Für Linie 01 wurden zwischen dem Fleischanteil nach MRI und dem Fleischanteil nach der Gruber- bzw. Bonner-Formel mit r = 0,67 bzw. r $=0,74$ niedrigere Beziehungen gefunden.

Tabelle 5

Korrelationen zwischen dem mit MRI und anhand von Schlachtkörpermaßen bestimmten Fleischanteil im Bauch (Correlations between lean content in belly determined by MRI and from carcass traits)

\begin{tabular}{lcccc}
\hline & MR-Imaging & Gruber-Formel & Bonner-Formel & BHZP-Formel \\
\hline MR-Imaging & 1 & 0,67 & 0,74 & 0,78 \\
Gruber-Formel & 0,82 & 1 & 0,85 & 0,88 \\
Bonner-Formel & 0,81 & 0,84 & 1 & 0,88 \\
BHZP-Formel & 0,87 & 0,88 & 0,91 & 1 \\
\hline Korrelationskoeflizienten oberhalb der Diagonalen gelten fur Linic 01, unterhalb für Linie 03
\end{tabular}

In Tabelle 6 sind die Ergebnisse der Varianzkomponentenschätzung aufgeführt. Der höchste Vatereinfluß war im Fleischanteil des Bauches zu verzeichnen. Für die Seitenspeckdicke und die Fleischfläche wurden niedrigere Intraklasskorrelationen berechnet; die niedrigste Varianz zwischen den Vätern wurde für die Merkmale Rückenspeckdicke, Fettfläche und Fleischanteil nach FOM gefunden.

Tabelle 6

Varianzkomponenten und Intraklasskorrelationen für Schlachtkörpermerkmale und Bauchfleischanteil (Variance components and intraclass correlation for carcass traits and lean content of the belly)

\begin{tabular}{lrcc}
\hline & $\mathrm{V}_{\mathrm{e}}$ & $\mathrm{V}_{\mathrm{s}}$ & IKK \\
\hline FA Halfte (FOM) & 10,19 & 1,41 & 0,12 \\
Rückenspeck & 8,79 & 0,82 & 0,09 \\
Seitenspeck & 13,16 & 4,35 & 0,25 \\
Fleischfläche & 10,46 & 2,45 & 0,19 \\
Fettilliche & 9,26 & 1,14 & 0,11 \\
FA Bauch (MRI) & 16,21 & 7,53 & 0,32 \\
\hline
\end{tabular}


4. Diskussion

Aufgrund von Besonderheiten bei der Vermarktung wird von Wissenschaft und Praxis eine objektivere Bewertung des Teilstückes Bauch angestrebt. Währende fette Bäuche fast ausschließlich für die Verarbeitung genutzt werden, eignen sich magere Bäuche vor allem in den Sommermonaten als Ladenware (Grillfleisch). Hierdurch können sich Preisunterschiede von über 20 DM pro Schwein ergeben (PFUHL und GLODEK, 1996). Da die Bezahlung von Schlachtkörpern für eine noch gezieltere Sortierung und Verwertung zukünftig vermehrt auf der Basis von Teilstücken erfolgen wird, kommt der genauen Kenntnis der Zusammensetzung von Schlachtkörpern auch auf der Zuchtstufe immer mehr Bedeutung zu. Voraussetzung für die züchterische Verbesserung einzelner Teilstücke ist eine zuverlässige Bestimmung ihrer geweblichen Zusammensetzung. Wie frühere Untersuchungen von BAULAIN et al. (1998) gezeigt haben, ist das nicht-invasive Magnet-Resonanz-Imaging ein geeignetes Verfahren, die Zusammensetzung von Schweinebäuchen zu erfassen. Wie bei der grobgeweblichen Zerlegung kommt bei dieser Methode keine Schätzformel zur Anwendung, da Muskelund Fettvolumina direkt am Teilstück gemessen werden können. Im Unterschied zur Zerlegung werden die Bäuche bei diesem Verfahren jedoch nicht „zerstört”, so daß eine weitere Vermarktung möglich ist.

Die Ergebnisse der vorliegenden Studie zeigen, daß sich die beiden BHZP-Mutterlinien in der Bauchzusammensetzung deutlich unterscheiden. Die Mittelwerte von $45,7 \%$ und $52,8 \%$ für die Linien 01 und 03 liegen dabei mehr als eine Standardabweichung auseinander. Zum Vergleich wiesen die ebenfalls mit MRI untersuchten Bäuche von Börgen bayerischer Mutterlinien (DLS) einen Fleischanteil von 48,6\% auf (BAULAIN et al., 1998). Auch die Betrachtung der Schlachtkörpermerkmale zeigt, daß die Börge der Mutterlinie 01 deutlich fetter waren als die der Linie 03. Dies gilt jedoch nicht für das Merkmal Rückenspeckdicke, wo eine Differenz von einem Millimeter nicht statistisch abgesichert werden konnte.

Die Korrelationen zwischen den in der Leistungsprüfung vergebenen Bauchpunkten und dem mittels MRI bestimmten Fleischanteil zeigen, daß eine Bonitierung, d. h. eine subjektive Beurteilung von Schweinebäuchen nur bedingt zur Beschreibung des Bauchfleischanteiles geeignet ist. Während eine Differenzierung zwischen den Linien möglich scheint, ist eine Bewertung von Bäuchen innerhalb der Linien wenig erfolgversprechend.

Gemäß den ALZ-Richtlinien von 1997 kann bei der Durchführung der stationären Geschwister-/Nachkommenprüfung der Fleischanteil im Bauch mit Hilfe von Schätzformeln, in die verschiedene Schlachtkörpermaße eingehen, bestimmt werden (THOLEN et al., 1998). Die Anwendung der für Mutterlinien gültigen Formel auf die Tiere des BHZP ergab, daß die Korrelation zu dem mit MRI bestimmten Muskelfleischanteil für Linie 03 mit $r>0,8$ relativ hoch, für Linie 01 mit $r<0,7$ deutlich niedriger war. Der Vergleich der Mittelwerte der mit den unterschiedlichen Methoden bestimmten Fleischanteile ergab sehr stark differierende Werte. Es ist hierbei jedoch zu berücksichtigen, daß dieser Vergleich nur mit Einschränkungen möglich ist, da die aus dem MRI resultierenden Werte die Einheit Vol.-\%, die auf Zerlegeergebnissen basierenden 
Schätzfunktionen jedoch die Einheit Gew.-\% haben. Eiree Umrechnung der Volumina in die entsprechenden Gewichte wurde an dieser Stelle nicht vorgenommen, da keine Meßwerte für die notwendigen spezifischen Gewichte von Muskel- und Fettgewebe vorlagen. Die Ergebnisse weisen jedoch darauf hin, daß die Gruber Formel insbesondere für die Linie $01 \mathrm{zu}$ einer Überschätzung des Fleischanteiles geführt hat. Ebenso weist die stark reduzierte Varianz des Fleischanteiles darauf hin, daß die Anwendung dieser Formel furr die Mutterlinien des BHZP zu unbefriedigenden Ergebnissen geführt hat. Die zum Vergleich durchgeführte Schätzung mit der bis 1997 in der Leistungsprüfung gültigen Bonner Formel zeigte dagegen eine bessere Übereinstimmung mit den Werten aus dem MR-Imaging. Die eigene, an den in diese Untersuchung einbezogenen Daten entwickelte Schätzformel zeigt erwartungsgemäß die beste Schätzung, konnte aber nicht an einer Validierungsstichprobe überprüft werden. Es stellt sich hier die Frage, ob eine herkunftsübergreifende Schätzung des Bauchfleischanteiles anhand von Muskel- und Fettmaßen des Schlachtkörpers immer möglich ist. Es ist denkbar, daß in der vorliegenden Studie Typunterschiede zwischen den BHZP- und den DLSSchweinen, an denen die Gruber Formel entwickelt wurde, Ursache für die relativ geringe Übereinstimmung waren.

In diesem Zusammenhang sollte auch bedacht werden, daß sich der Muskelfleischanteil im Bauch durch die Hinzunahme von direkt am Bauch erhobenen Maßen besser schätzen läßt als allein über Muskel- und Fettmaße des Rückens (BAULAIN et al., 1998). Die Frage nach einer objektiven Bauchbewertung in der Leistungsprüfung ist nach wie vor nicht endgültig geklärt und wird in einer z. Z. laufenden Studie weiter bearbeitet.

Ein Ziel dieser Untersuchung war, durch die gezielte Auswahl der Väter und Nachkommen einen Anhaltswert für die Varianz zwischen Vätern zu erhalten. Auch unter Berücksichtigung der geringen Tierzahl und der damit verbundenen Überschätzung der Intraklasskorrelationen deutet sich an, daß im Bauchfleischanteil eine größere Variation zwischen Vätern vorhanden ist als bei den anderen in der Leistungsprüfung erhobenen Schlachtkörpermerkmalen. Dies gilt insbesondere für die Rückenspeckdicke, die im Rahmen der Selektion auf Fleischfülle über Ultraschallmessungen schon lange intensiv züchterisch bearbeitet wird. Eine gesonderte Rangierung der Väter nach dem Bauchfleischanteil könnte ein geeignetes Mittel sein, den Bauch gezielt züchterisch zu bearbeiten.

\section{Literatur}

ALZ:

Richtlinie für die Stationsprüfung auf Mastleistung, Schlachtkörperwert und Fleischbeschaffenheit beim Schwein. Ausschuß für Leistungsprüfungen und Zuchtwertfeststellung beim Schwein (1997) BAULAIN, U.:

Magnetic resonance imaging for the in vivo determination of body composition in animal science. Computers and Electronics in Agriculture 17 (1997), 189-203

BAULAIN, U.; HENNING, M; THOLEN, E.; WITTMANN, W.; PESCHKE, W.:

Objektive Erfassung des Fleischanteils im Schweinebauch. 2. Mitteilung: Verwendung von Bildinformationen aus dem MR-Imaging. Züchtungskunde, Stuttgart 70 (1998), 205-212 
BRANSCHEID, W.; DOBROWOLSKI, A.; HÖRETH, R.:

Bestimmung der Handelsklassen und des Handelswertes von Schweinehälften mit dem Gerät Autofom. Fleischwirtschaft, Frankfurt/M. 77 (1997), 619-622

BRONDUM, J.; EGEBO, M.; AGERSKOV, C.; BUSK, H.:

On-line pork carcass grading with the Autofom ultrasound system. J. Anim. Sci., Champaign, III. 76 (1998), 1859-1868

FEWSON, D.; BRANSCHEID, W.; SACK, E.:

Untersuchungen über den Fleischanteil einzelner Teilstücke und der Schlachthälfte beim Schwein. Züchtungskunde, Stuttgart 62 (1990), 38-51

PFUHL, K; GLODEK, P.

Die Bestimmung des Fettgehaltes von Schweinebäuchen mittels NIR und dessen Beziehung zu anderen SAS INSTITUTE INC.

SAS/STAT User's Guide, Version 6, Fourth Edition, Vol. 2, Cary, NC, 1989, 846 pp.

THOLEN, E.; PESCHKE, W.; BAULAIN, U.; SCHELLANDER, K.:

Objektive Erfassung des Fleischanteils im Schweinebauch. 1. Mitteilung: Entwicklung von Schäłzgleichungen aus Schlachtkörpermaßen. Züchtungskunde, Stuttgart 70 (1998), 196-204

Eingegangen: 29.09 .1999

Akzeptiert: 12.10 .1999

Anschriften der Verfasser

Dr. ULRICH BAULAIN

Institut für Tierzucht und Tierverhalten der FAL, Mariensee

Höltystraße 10

D-31535 Neustadt

Dr. HUBERT HENNE

Züchtungszentrale Deutsches Hybridschwein GmbH,

Soltauer Str. 89

D-21335 Lüneburg 